\title{
A Circular Needle Path Planning Method for Suturing in Laparoscopic Surgery
}

\author{
Florent Nageotte, Philippe Zanne, Michel de Mathelin and Christophe Doignon \\ LSIIT (UMR 7005 CNRS), Louis Pasteur University, Strasbourg, France \\ Parc d'Innovation, bd S. Brant 67400 Illkirch \\ e.mail : nageotte@eavr.u-strasbg.fr
}

\begin{abstract}
The work presented in this paper addresses the problem of the stitching task in laparoscopic surgery using a circular needle and a conventional 4 DOFs needle-holder. This task is particularly difficult for the surgeons because of the kinematics constraints introduced by the trocar. So as to assist the surgeons, we propose to compute possible pathes for the needle through the tissues, which limits as much as possible tissues deformations while driving the needle towards the desired target. The article proposes a kinematic analysis and a geometric modeling of the stitching task. Based on this, some simple but useful information can be obtained to help the surgeon. The description of the task with well-chosen state variables allows to simply express the path planning problem. Conditions for the existence of acceptable pathes are given and a method to compute possible pathes is presented. Resulting pathes are shown to be satisfactory even under awkward configurations.
\end{abstract}

Index Terms-laparoscopic surgery, path planning, geometrical modeling, kinematic constraint, computer-aided surgery

\section{INTRODUCTION}

Suturing is probably one of the most difficult tasks for surgeons in laparoscopic surgery, as shown by Cao et al. [1]. Stitching, the motion that makes the needle go through tissues is the first stage of suturing, before knot tying. Most stitching tasks involved in surgery can be described as follows. The surgeon first selects an entry point and an exit point on the surface of the tissues, on each side of the lesion. He next drives the tip of the needle to the entry point, pierces the tissue, drives the tip of the needle to the exit point, and then bites the tissue a second time. The needle can then be released, caught again close to the tip, and pulled out of the tissue.

Reaching the exit point may be a difficult sub-task because it must be realized without direct visual feedback. In laparoscopic surgery the task becomes even more difficult because of the kinematics and visual constraints induced by the laparoscopic setup. With conventional needle-holders, the possible movements of the needle are restricted to only four degrees of freedom (DOFs) by the trocar constraint (see figure 1). Moreover, the monocular vision provided by the endoscopic camera makes the estimation of distances and angles very difficult. Consequently, the surgeon cannot simply figure out which motions of the needle are feasible The surgeon must often proceed by trial and, in many cases, the desired exit point can only be reached at the expense of large and undesirable deformations of tissues.
In order to improve the dexterity of the surgeon, recent surgical robotic systems, like Zeus from Computer Motion or DaVinci from Intuitive Surgical, have provided instruments with supplementary degrees of freedom. But these devices are expensive, and the time required to set up the system in the operating room can critically increase the duration of the interventions. Moreover, the motions of the needle can be complex even with 5 or 6DOFs effectors.

An other direction has been to develop new specific instruments for suturing. Stitching machines, like Endostitch from US Surgical, allow to simply drive a straight needle through tissues without needing to release the needle. However such instruments cannot be used in all kind of suturing.

Some systems try to help the surgeon by providing him information which he cannot obtain under usual conditions, like stereo endoscopes used together with head-mounted $3 \mathrm{D}$ visualization devices. However this does not solve the basic problem for stitching, which is the difficulty to know the possible positions and motions of the needle.

Virtual training systems have also been developped in order to provide realistic and low-cost environments to the surgeons. For example, LeDuc et al.[8] have simulated the interaction between suture and tissues. Ladd et al.[6] have also proposed knot tying simulation. But these works do not intend to bring additionnal information to the surgeon.

Finally, there are works towards robot assisted tasks. Krupa et al [5] have developped an autonomous system for leading the surgical instruments in the field of view of the endoscopic camera. Kang et al.[4] work on an autonomous knot tying system, but, to our knowledge, nothing has been made yet concerning the stitching step.

The work presented here is a first modeling step which aims at providing help to the surgeons, by proposing suitable pathes for the needle through the tissues, which minimize the tissues movements and deformations. This article presents the geometric and kinematic analysis of the task The outline of this work, the objectives and some possible applications of the modelling have been briefly described in [9].

The following of the paper is divided into four sections. In the first section, we present a geometrical and kinematics model of the stitching task with a conventional 4DOFs instrument. Then, in the second section, we show the information that can be deduced from the previous analysis. The third section presents a path planning method 


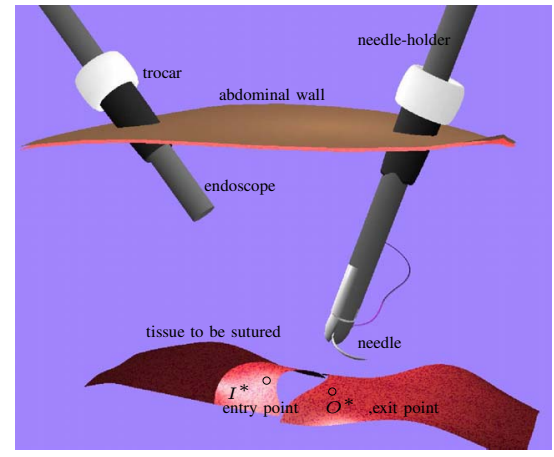

Fig. 1. Outline of the laparoscopic setup with desired entry point $\left(\mathrm{I}^{*}\right)$ and exit point $\left(\mathrm{O}^{*}\right)$

for the needle, that allows stitching with minimal tissue deformation. Discussions about current and future work will be found at the end of the paper.

\section{ANALYSIS OF THE STITCHING TASK WITH A CONVENTIONAL NEEDLE-HOLDER AND A CIRCULAR NEEDLE}

\section{A. Description of the laparoscopic setup}

In laparoscopic surgery, small incisions are made into the abdominal wall of the patient. Trocars are then introduced into these incisions to allow surgical instruments and endoscopes to go through (c.f. figure 1).

Because of the trocars, the mobility of the instruments is reduced. This work deals with conventional needle holders. These are equipped with jaws to grasp the needle, which are rigidly linked to the cylindrical body of the instrument. This instrument has then only 4DOFs which can be described by a spherical joint on the trocar center $Q$, and a concurrent prismatic joint.

The geometrical study of the stitching task presented afterwards has been made under the conditions used in training devices. The movements of the tissues to be sutured, mainly due to respiration will not be considered. Tissues to be sutured are thin and can be locally approximated by a planar surface around the suturing area. Hereafter, this plane will be called the stitching plane. Tissues can deform under the action of the needle or the needle-holder, but we are only interested in the study of the movements of the tissue in the stitching plane.

Finally, there are no obstacles in the workspace.

\section{B. Modeling of the stitching task}

The stitching task is the movement of the needle that allows to go through the tissue from an entry point $I^{*}$ to an exit point $O^{*}$ on the other side of the tissue. The task can be modeled as a path planning problem between two reference points given by the surgeon. Hereafter we will call initial position a position of the needle for which its tip is in $I^{*}$, and final position a position of the needle for which its tip is in $O^{*}$. The ideal movement is to pierce the tissue in $I^{*}$ and reach $O^{*}$ without deforming or tearing the tissues. It is also desirable that initial and final positions

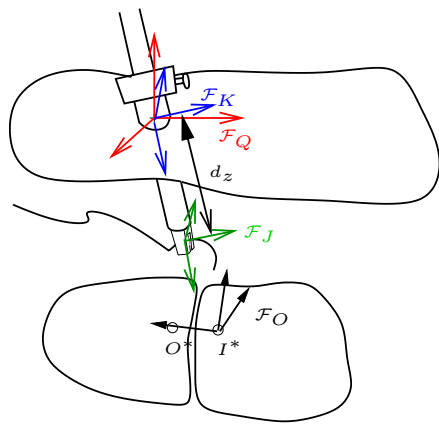

Fig. 2. locations of the frames used in the geometrical modeling of the system

of the needle allow to bite the tissue correctly, i.e. that the tangent to the tip of the needle be close to the normal to the tissue.

\section{Notations and definition of useful frames}

Hereafter we use the following notations :

$\mathcal{F}_{I}$ denotes a frame of axis $\left(x_{I}, y_{I}, z_{I}\right)$ centered on point I. $T_{i j}$ and $R_{i j}$ are the translation and the rotation between frames $\mathcal{F}_{I}$ and $\mathcal{F}_{J} . R_{x}(\theta)$ is a rotation of angle $\theta$ around the axis $x .{ }^{l} I J$ is the vector from point $I$ to point $J$ expressed in $F_{L}$.

Frames have been attached to the different elements involved in the stitching task and are shown on figures 2 and 3.

$\mathcal{F}_{O} \quad$ is attached to the organ, centered in $I^{*}$, with $z_{O}$ along the normal to the tissue and $x_{O}$ along $I^{*} O^{*}$.

$\mathcal{F}_{Q} \quad$ is the frame attached to the patient with its origin on the trocar center $Q$.

$\mathcal{F}_{J} \quad$ is attached to the instrument at the center of the jaws with $z_{J}$ along the axis of the instrument. $x_{J}$ is parallel to the jaws.

$\mathcal{F}_{K} \quad$ is attached to the trocar, centered in $Q$, and $R_{k j}=$ $\mathcal{I}_{3}$ and $T_{k j}=\left(0,0, d_{z}\right)^{T}$.

$\mathcal{F}_{N} \quad$ is the frame for the needle. Its origin is the center of the needle and it is oriented as shown on figure 3.

With conventional needle-holders the surgeon can control four DOFs : ${ }^{k} \omega_{k / q}=\left(\omega_{x}, \omega_{y}, \omega_{z}\right)^{T}$, and ${ }^{k} V_{j / k}^{J}=$ $\left(0,0, v_{z}\right)^{T}$. The state of the instrument can be described by the set of Euler angles $\theta_{x}, \theta_{y}, \theta_{x}$ and $d_{z}$ with

$$
\begin{aligned}
R_{q k} & =R_{x}\left(\pi+\theta_{x}\right) R_{y}\left(\theta_{y}\right) R_{z}\left(\theta_{z}\right) \\
T_{k j} & =\left(0,0, d_{z}\right)^{T}
\end{aligned}
$$

The interaction between the jaws of the needle-holder and the needle is considered as punctual. The pose of the needle in the needle-holder can then be defined using four parameters (see figure 3): $\beta$, the angular position of the jaws on the needle, angles $\zeta$ and $\psi$ which control the orientation of the needle in the needle-holder, and $b$ the position of the needle in the jaws. This parameter does not actually act on the stitching, since a $\Delta_{b}$ variation of $b$ can be compensated by using $d_{z}+\Delta_{b}$ instead of $d_{z}$. 


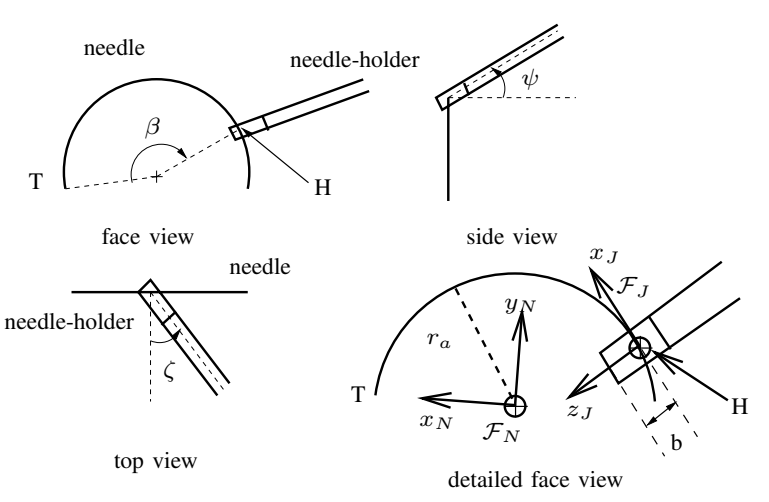

Fig. 3. Parameters for the needle handling

Then, without loss of generality, it can be considered that the needle is in the center of the jaws and the handling point on the needle $H$, and $J$, the center of the jaws, are superimposed. $\beta, \zeta, \psi$ are called the handling parameters.

This manner, using an Euler decomposition

$$
\begin{aligned}
R_{n j} & =R_{z}\left(\beta-\frac{\pi}{2}\right) * R_{x}(\psi) * R_{y}(\zeta) \\
T_{n j} & =r_{a} *(\cos \beta, \sin \beta, 0)^{T}
\end{aligned}
$$

All the possible orientations of the needle are described by $\zeta \in]-\frac{\pi}{2}, \frac{\pi}{2}\left[\right.$ and $\psi \in\left[-\frac{\pi}{2}, \frac{3 \pi}{2}\right]$.

\section{Expressing the state of the system around a fixation point}

When the needle is held by the needle-holder, any point $N$ of the needle can reach any position $M$ of the abdomen of the patient using only $\omega_{x}, \omega_{y}$, and $v_{z}$. When using the fourth DOF, $\omega_{z}$, it is possible to give different orientations to the needle while maintaining $N$ in $M$.

The possible movements when $N$ is in $M$ is an axial rotation around $Q M$ (see figure 4). Indeed, the rigid object composed by the needle-holder and the needle has a 3DOFs kinematics constraint in $M$ (point on point constraint) and a 2DOFs constraint in $Q$ (straight line on point constraint). Hence these constraints generally allow a unique free DOF for the needle/needle-holder set. Actually the constraints enforce the point of the needle-holder that must lie in $Q$ and the unique free movement is the rotation around the axis $Q M$. Consequently, every point $P$ of the needle or the needle-holder moves on a circle centered on the axis $Q M$. Hereafter this circle will be called the motion circle for $P$ and the point $M$ a fixation point for $N . N$ is the fixed point.

Let us interest in the properties of these circles. We call $\mathcal{F}_{M}$ the frame centered in $Q$, with $z_{M}$ pointing towards $M$ (see figure 4)).

The rotation $R_{m j}$ can be written using an Euler decomposition

$$
R_{m j}=R_{z}(\rho) * R_{y}(\chi) * R_{z}(\kappa)
$$

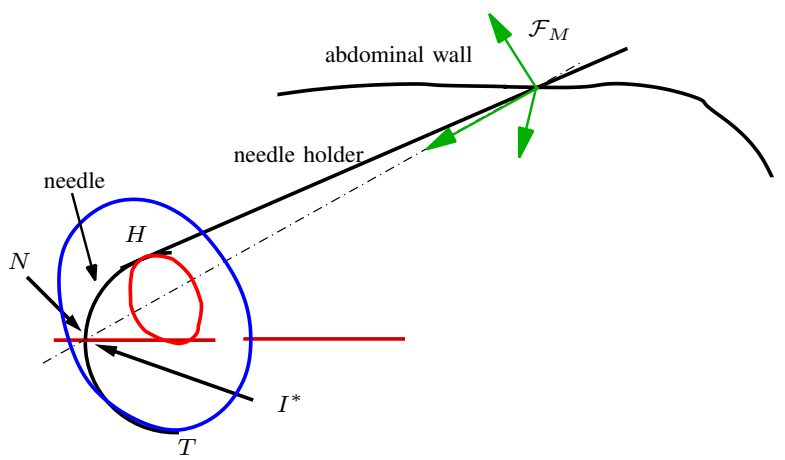

Fig. 4. Motion circles for the handling point $H$ and the tip of the needle $T$ when $I^{*}$ is a fixation point for $N$

and one can show that

$$
\begin{aligned}
\kappa & =-\arctan \frac{Y_{N}}{X_{N}} \text { if } X_{N} \neq 0, \frac{\pi}{2} \text { otherwise } \\
\chi & =\arcsin \frac{Y_{N}}{Z_{M} * \sin \kappa} \\
d_{z} & =Z_{M} * \cos \chi-Z_{N}
\end{aligned}
$$

where $\left(\begin{array}{c}X_{N} \\ Y_{N} \\ Z_{N}\end{array}\right)$ is the position of $N$ in the frame $\mathcal{F}_{J}$. By defining $N$ by its angular position with respect to the tip of the needle, $\varphi_{N}$, this position can be computed from the handling parameters

$$
{ }^{j} H N=R_{j n} *\left(\left(\begin{array}{c}
r_{a} * \cos \varphi_{N} \\
r_{a} * \sin \varphi_{N} \\
0
\end{array}\right)-T_{n j}\right)
$$

One can also compute the radius and the position of the circle for $H$

$$
\begin{aligned}
r_{H} & =d_{z} *|\sin \chi| \\
{ }^{m} Z_{J} & =d_{z} * \cos \chi
\end{aligned}
$$

With $\chi, \kappa$ and $\rho$, the position of the needle-holder and the needle are completely defined and it is possible to compute the properties of the motion circle of any other point of the needle.

$\psi=\frac{\pi}{2} \pm \pi$ is a particular configuration that cannot be described by the previous equations. The axis of the needleholder is then in the plane of the needle and $Y_{N}=0$. In such cases $\kappa=0$ and $\chi=-\arcsin \frac{X_{N}}{Z_{M}}$. Practically, these cases are of little interest, because $\omega_{z}$ does not allow the rotation of the needle on its axis anymore. These cases will not be considered in the following of the article.

As a conclusion, the state of the needle can be completely described by a fixation point, a fixed point defined by $\varphi_{N}$ and the angle $\rho$. From this description it is possible to obtain the usual parameters $R_{q k}$ and $T_{k j}$ from the following equations

$$
\begin{aligned}
R_{q k} & =R_{q m} * R_{m j} \\
T_{k j} & =R_{q k}^{T} *{ }^{q} Q M-{ }^{j} H N
\end{aligned}
$$

Most of the following results are based on this interesting property. 


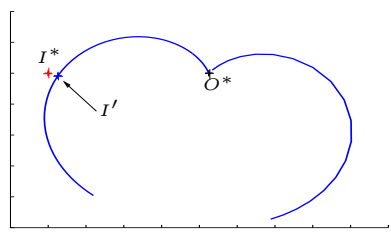

Fig. 5. Curve of the possible entry points on the stitching plane with given $O^{*}$ and $I^{*} . I^{\prime}$ is the closest point of the curve to $I^{*}$.

\section{SOLVING STITCHING PROBLEMS WITH THE MODEL}

Using this model of the stitching allows to simply solve several problems.

a) Finding final positions: From nown on, it is assumed that the handling parameters and the position of the trocar in $\mathcal{F}_{O}$ are known.

Given a desired exit point, one can determine the possible entry points $O^{*}$ for the tip of the needle.

$O^{*}$ is a fixation point for the tip of the needle. Using equations $(6-8)$, one can compute $\chi, \kappa$ and $d_{z}$. The position of the needle and the needle-holder can then be obtained for sampled values of $\rho$. For each position, the intersection of the needle and the stitching plane is computed and expressed in frame $\mathcal{F}_{N}$. If there exists an intersection for an angular position in $[0-\beta]$ then the intersection, is a possible entry point. Otherwise there is no entry point for the given position of the needle. Figure 5 shows a curve for possible entry points.

The minimal distance from $I^{*}$ to the curve of possible entry points, is an estimation of the minimum required deformation of the tissue, to reach $O^{*}$ by entering in $I^{*}$.

b) Computing piercing angles: In the same manner, it is possible to compute the possible initial positions of the needle for a given entry point $I^{*}$. Then, $I^{*}$ is a fixation point for the tip of the needle and initial positions are obtained by sampling $\rho$. For the initial and final positions, we define the piercing angle $\mathcal{P} \mathcal{A}$, the angle between the tangent to the tip of the needle and the normal to the tissue at the piercing point. It can simply be computed by

$$
\mathcal{P} \mathcal{A}=\arccos (n . t)
$$

where $n$ is the normal vector to the stitching plane and $t$ the tangent to the tip of the needle.

Practically, the choice of the initial and final positions for the needle can be based on the minimization of a cost for each position. For the initial position the cost is a trade-off between the piercing angle and the distance of the handling point from the tissue. For the final position, the piercing angle must be considered together with the deformation distance.

It will be shown in the next section that finding possible initial and final positions does not guarantee the existence of a path.

\section{PATH PLANNING FOR THE NEEDLE}

Once a needle handling pose has been fixed and acceptable initial and final positions found, the problem is to find a feasible path between these two positions. In this section we present a method to find an acceptable path which minimizes the longitudinal deformation of the tissue.

\section{A. Initial problem}

Knowing the minimal deformation of the tissue, given by the distance between $I^{*}$ and $I^{\prime}$, the path existence problem can be defined as finding successive positions of the needle for which the tip of the needle $T$ is under the stitching plane, the handling point $H$ above the stitching plane, and for which the needle goes through the tissue in an area around $I^{*}$ and $I^{\prime}$. This area could for instance be defined as a circle of radius $\left\|I^{*} I^{\prime}\right\|$ centered in $I^{*}$. The 4D configuration space of the needle and the instrument, is then very cluttered. Under such conditions, global path planning methods are not recommended and local methods, like potential methods, are preferred. Unfortunately these methods show a lot of local minima in this problem.

That is why we propose to simplify the path planning problem the following manner.

\section{B. Path planning without deformation}

1) Path existence: It is assumed in this section that $I^{*}$ and $I^{\prime}$ are identical We then wish to find, if it does exist, a path for the tip of the needle from $I^{*}$ to $O^{*}$ that does not involve longitudinal movement or deformation of the tissues. In other words the entry point is considered as a punctual hole in a rigid surface through which the needle must be lead to $O^{*}$. Under these conditions, the configuration of the system can be expressed in a $2 \mathrm{D}$ configuration space with the state variables $\varphi_{n}$ and $\rho$ where $\varphi$ is the angular position of the point $N$ of the needle that is in $I^{*}$, and $\rho$ the angular position of the tip of the needle on its motion circle (see figure 4).

The existence of a path can be expressed :

$$
\forall \varphi_{n} \in[0-\alpha], \exists \rho / z_{H}>0 \text { and } z_{T}<0
$$

where $z_{H}$ and $z_{T}$ the positions of the handling point and the tip of the needle w.r.t. the stitching plane.

$\alpha$ is the angular position with respect to the tip of the needle of the point of the needle which is in $I^{*}$ when the tip is in $O^{*}$ and is defined by

$$
\alpha=\pi-2 \arccos \frac{\left\|I^{*} O^{*}\right\|}{2 r_{a}}
$$

The existence does not depend, neither on the orientation of the needle in the initial and final positions, nor on the position of the exit point. It only depends on the position of the trocar with respect to the stitching plane $T_{O Q}$ and on the stitching distance $\|I O\|$.

The path existence can be established numerically by sampling $\varphi_{n}$. For every value $\left.\varphi_{n} \in\right] 0-\alpha[$, the existence of $\rho$ so that $z_{H}>0$ and $z_{T}<0$ is determined analytically. 
2) Path generation: General path planning problems can be solved using two main kinds of methods : local methods and global methods. For an overview of classical methods, see the reference work of Latombe [7].

We chose to use a discrete complete method. The configuration space is discretized by sampling $\varphi_{n}$ and $\rho$. With only two degrees of freedom, the computation of a large number of configurations has a moderate cost. With a small enough sampling step, a straight line in the configuration space is an acceptable local path to connect two configurations. The sampled values of $\varphi_{n}$ and $\rho$ form a 2D map. The free configurations and the obstacles are determined by computing for each cell of the map the position of the handling point and the tip of the needle. Configurations for which $z_{T}>0$ or $z_{H}<0$ are then forbidden.

Qualitatively, a path for the needle is good when the motions of the needle are smooth and when the tip of the needle goes deeply under the stitching plane. Indeed, a path for which the tip moves nearby the surface of the tissue is neither natural, nor desirable.

So as to enforce a path with deep penetration under the tissue we associate a cost with each position in the map of the following empiric form

$$
\cos t= \begin{cases}\frac{K}{1-Z_{T}}-D & \text { if } Z_{T} \in\left[-\frac{r_{a}}{2} ; 0\right] \\ 0 & \text { if } Z_{T}<-\frac{r_{a}}{2} \\ \infty & \text { if } Z_{T}>0\end{cases}
$$

with high values when the tip of the needle is close to the stitching plane.

Then, we define the possible neigbours of any cell $\left\{\varphi_{n}, \rho_{i}\right\}$ of the map. They are the closest configurations, in the sense of the distance given in eq. 17, for which $\varphi=$ $\varphi_{n+1}$, and the configurations $\left\{\varphi_{n}, \rho_{i+1}\right\}$ and $\left\{\varphi_{n}, \rho_{i-1}\right\}$, taking into account the circularity of $\rho$. This manner, the "return" of the needle is impossible and the map becomes an oriented graph.

The overall cost associated with a path in the graph is computed as the wheighted sum of the configuration costs of the nodes that constitute the path, and the distance between two successive configurations. The distance between two configurations $C_{n}$ and $C_{n+1}$ is defined by

$$
\text { dist }=\max \left(\left\|H_{n} H n+1\right\|,\left\|T_{n} T n+1\right\|\right) .
$$

The optimal path with respect to the proposed cost function is computed using an algorithm based on the historical algorithm proposed by Dijkstra [2].

\section{Path planning in general cases}

In the general cases $I^{\prime}$ is different from $I^{*}$. We then choose to enforce the trajectory of the entry point along the path. For example one can use a straight line from $I^{*}$ to $I^{\prime}$ : this way the minimal deformation of the tissue is guaranteed.

When the trajectory of $I$ is chosen, the state of the needle can be described by the fixation point $I$, the fixed point defined by $\varphi$ and the value of $\rho$. It is then possible to use
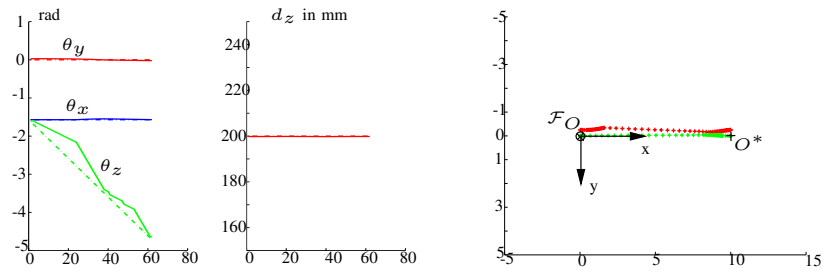

Fig. 6. Left : "Temporal" outline of the generated (solid) and expected (dashed) trajectory in function of $\varphi$ in a simple case Right : Positions of the handling point (red) and the tip of the needle (green) along the path seen normaly to the stitching plane.

the same path planning method as proposed previously for the particular case.

The method ensures to find the optimal path with respect to the defined cost function. However the found path is optimal only with respect to the chosen sampling on $\varphi$ and $\rho$ and resampling can consequently modify the resulting path. Despite these inconveniences the generated pathes are satisfying.

\section{Simulation results}

For the following simulations, the radius of the needle is $r_{a}=5 \mathrm{~mm}$ and all lengthes are given in millimeters. The sampling resolution is sixty values for $\varphi_{n}$ and hundreds values for $\rho$, which has been empirically shown to be sufficient.

So as to validate the proposed method, the computed pathes has been compared with pathes that would be manually and naturally chosen. A special configuration has been considered, for which the ideal movement of the needle can easily be found. It is described by the following parameters : $\zeta=0, \psi=0, \beta=\pi, d_{I O}=10$ and $T_{O Q}=(5,-200,0)^{T}$. This configuration is not realistic, since the trocar lies in the stitching plane. The axis of the needle-holder is normal to the plane of the needle. Since $\left\|T_{O Q}\right\| \gg r_{a}$, the rotation of the needle around its axis is almost feasible.

The expected reference trajectory is then circular, planar, without deformation of the tissues and with $\theta_{z}$ varying from $-\frac{\pi}{2}$ to $-3 \frac{\pi}{2}$. The results are presented on figure 6 . The path is almost planar as shown by the position of the handling point $H$ and the tip of the needle $T$ along the trajectory. The variations of $\theta_{x}, \theta_{y}$ and $d_{z}$ are very small as expected and the obtained results can be considered as satisfactory. However the evolution of $\theta_{z}$ with time does not have the wished outline. There are two reasons for this : first, the nodes of the oriented graph are not equally spaced; next, the choice of the cost bound with the nodes of the graph acts upon the outline of the path.

Moreover, it must be noted that the proposed method only generates a path, not a trajectory. The temporal following of the generated path should take into account the behaviour of the tissues during piercing and the normal deformations of the tissues. This is part of our work but out of the scope of this article. 

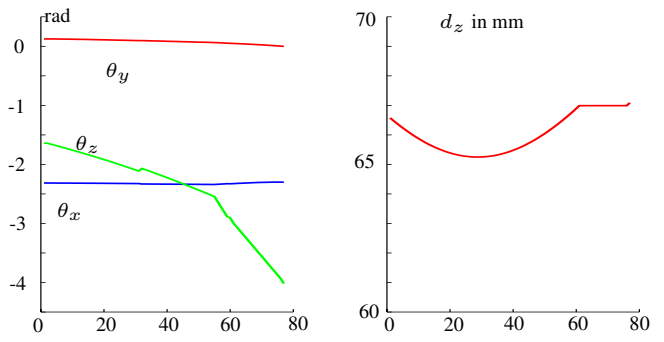

Fig. 7. Temporal evolution of the state of the needle holder. $\theta_{x}$ and $\theta_{y}$ are the angles around the trocar, $\theta_{z}$ the angle of the rotation around the axis of the needle-holder and $d_{z}$ the depth of the instrument in the abdomen
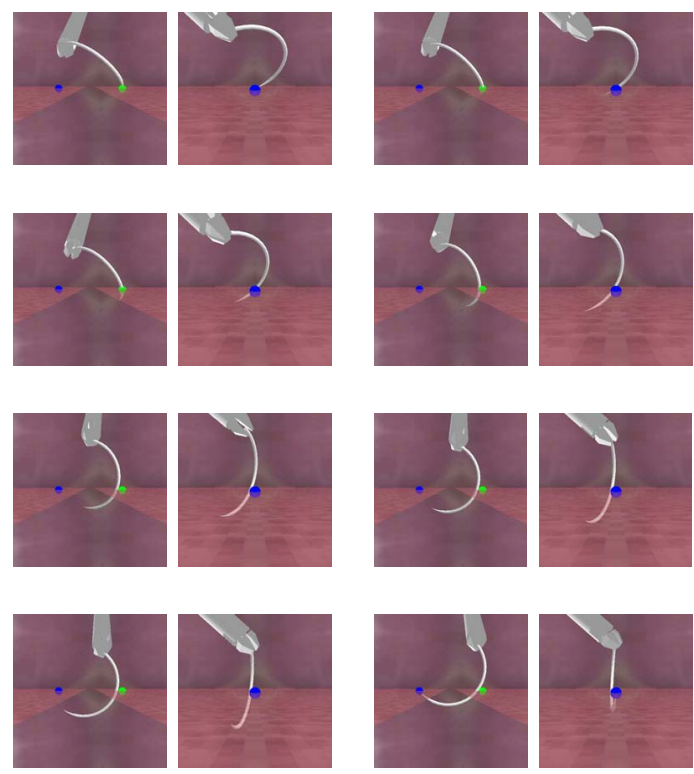

Fig. 8. Some computed positions of the needle along the generated path. The path was computed using 60 values for $\varphi$ and 100 values for $\rho$ giving rise to a 65 samples path. From top to bottom and left to right are the initial position, positions at steps $10,20,30,40,50,57$ and the final position given under two points of view. The green and blue points are the desired entry and exit points.

The goal of the second simulation is to show the ability of the proposed method to deal with complex cases. For this simulation the trajectory of $I$ has been chosen to be a straight line and the parameters are $\zeta=0.45, \psi=0.5$, $\beta=\pi, d_{I O}=8.5$ and $T_{O Q}=(0,-50,50)^{T}$.

Figure 8 shows sampled positions of the computed path and the corresponding temporal evolution of the state of the needle-holder is presented on figure 7 . In this case the deformation of the tissue is required to reach $O^{*}$. The initial position does not point towards $O^{*}$ and a complex "torsion" movement of the needle is required.

\section{E. Extension to non-planar cases}

Up to now, it has been assumed that the tissues could be locally approximated by planar surfaces. This is not always possible, for example in vessels anastomosis where tissues have important curvature. However, the presented methods can easily be extended to such cases as soon as a model of the tissues is available. The intersections between the needle and the tissues could then be computed numerically. In a first approach a simple cylindrical modeling of the vessels could be sufficient. The use of preoperatives data obtained by multi-modal images is a conceivable solution to improve the modeling. Systems based on laser projection techniques as proposed by Hayashibe et al. [3] could also be useful during the operation.

\section{CONCLUSION AND FUTURE WORK}

Based on a geometrical modeling of the stitching task we have proposed theoretical methods to help the surgeon before and during the stitching. With the knowledge of the position and orientation of the needle in the needleholder, it is possible to plan the possible exit points of the needle, the piercing angles and the deformations of the tissues that will be involved during stitching. We have proposed a method to plan an acceptable path for the needle even under complex conditions. In manual laparoscopic surgery, this path could then be shown to the surgeon by using augmented reality and be used as a reference. In robot-assisted suturing the path planning method could be used to propose automatic modes for stitching under the supervision of the surgeon.

On the practical level, the required information is obtained by the use of vision. Color endoscopic cameras used with marked needle and needle-holder allow to estimate the handling parameters and the position of the trocar. We also use a laser pointing device previously developped by Krupa et al[5] so as to get the reference points from the surgeon and estimate the stitching plane. We are currently implementing the path planning software on an Aesop surgical arm from Computer Motion.

\section{REFERENCES}

[1] C. Cao, L. MacKenzie, and S. Payandeh. Task and motion analyses in endoscopic surgery. In ASME IMECE Conference Proceedings : 5th Symposium on Haptic Interfaces for Virtual Environment and Teleoperator systems, pages 583-590, Atlanta, USA, 1996.

[2] E. W. Dijkstra. A note on two problems in connection with graphs. Numerische Mathematic, 1:269-271, 1959.

[3] M. Hayashibe, N. Suzuki, A. Hattori, and Y. Nakamura. Intraoperative fast $3 \mathrm{~d}$ shape recovery of abdominal organs in laparoscopy. In Proceedings of the 5th International Conference on MICCAI, pages 356-363, Tokyo, September 2002.

[4] H. Kang and J. Wen. Robotic knot tying in minimally invasive surgeries. In IEEE/RSJ International Conference on Intelligent Robots and Systems, Lausanne, Switzerland, 2002.

[5] A. Krupa, J. Gangloff, C. Doignon, M. de Mathelin, G. Morel, J. Leroy, L. Soler, and J. Marescaux. Autonomous 3-D positioning of surgical instruments in robotized laparoscopic surgery using visual servoing. IEEE Transactions on Robotics and Automation, 19(5):842853, October 2003.

[6] A. Ladd. Simulated knot tying. In IEEE International Conference on Robotics and Automation, Washington D.C., May 2002.

[7] J. C. Latombe. Robot Motion Planning. Kluwer Academic Publishers, 1991.

[8] M. LeDuc, S. Payandeh, and J. Dill. Toward modeling of a suturing task. In Graphics Interface, 2003.

[9] F. Nageotte, M. de Mathelin, C. Doignon, L. Soler, J. Leroy, and J. Marescaux. Computer-aided suturing in laparoscopic surgery. In Proceedings of the 18th International Congress on Computer Assisted Radiology and Surgery, Chicago, June 2004. 